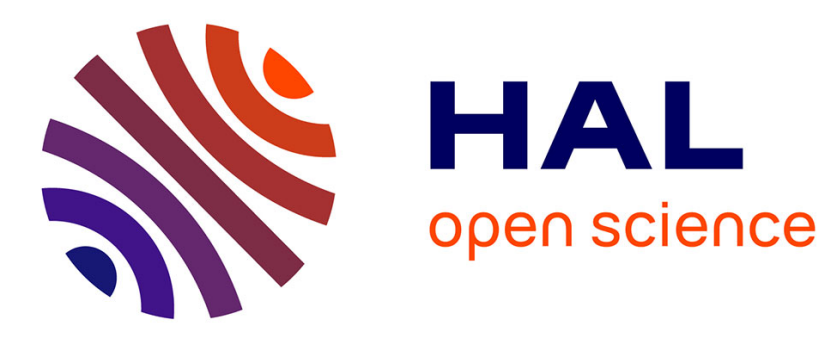

\title{
Multi-scale arithmetization of linear transformations
} Loïc Mazo

\section{To cite this version:}

Loïc Mazo. Multi-scale arithmetization of linear transformations. Journal of Mathematical Imaging and Vision, 2018, 61, pp.432-442. 10.1007/s10851-018-0853-6 . hal-01405461v3

\section{HAL Id: hal-01405461 \\ https://hal.science/hal-01405461v3}

Submitted on 6 Aug 2018

HAL is a multi-disciplinary open access archive for the deposit and dissemination of scientific research documents, whether they are published or not. The documents may come from teaching and research institutions in France or abroad, or from public or private research centers.
L'archive ouverte pluridisciplinaire $\mathbf{H A L}$, est destinée au dépôt et à la diffusion de documents scientifiques de niveau recherche, publiés ou non, émanant des établissements d'enseignement et de recherche français ou étrangers, des laboratoires publics ou privés. 


\title{
Multi-scale arithmetization of linear transformations
}

\author{
Loïc Mazo \\ ICube, University of Strasbourg, CNRS \\ 300 Bd Sébastien Brant - CS 10413 - 67412 ILLKIRCH, FRANCE
}

August 6, 2018

\begin{abstract}
A constructive nonstandard interpretation of a multiscale affine transformation scheme is presented. It is based on the $\Omega$-numbers of Laugwitz and Schmieden and on the discrete model of the real line of Reeb and Harthong. In this setting, the nonstandard version of the Euclidean affine transformation gives rise to a sequence of quasi-linear transformations over integer spaces, allowing integer-only computations.
\end{abstract}

\section{Introduction}

In the past decade, a series of papers [3, 6, 9, 17] was devoted to the the arithmetization of the continuum with the goal to solve numerical problems using only integers. In particular, a multi-scale arithmetization of the Euler scheme for solving differential equations was developed and used to produce discrete representations of continuous functions and curves. These works are rooted, on the one hand, in the studies of G. Reeb and J. Harthong on nonstandard analysis [8, 10] that led to a discrete model of $\mathbb{R}$, the Harthong-Reeb line. This model, though it requires to dispose of an infinitely large number (which was axiomatically assumed), allowed J.P. Reveillès [19, 20] to construct a discretization scheme that resulted in its seminal work on the digital line. On the other hand, D. Laugwitz and C. Schmieden [16,21] proposed a constructive definition of infinitely large numbers: the $\Omega$-numbers. Defining the Harthong-Reeb line with the $\Omega$-numbers was done in the $\mathrm{PhD}$ thesis of $\mathrm{A}$. Chollet [2] and gave rise to the $\Omega$-arithmetization scheme.

In the present paper, we aim at using the multi-scale discretization scheme of Chollet et al. to obtain a multiscale discretization of affine transformations between Euclidean spaces. In her PhD thesis [11], M.A. Jacob-Da Col, who was a student of Reveillès, studied the properties of an arithmetization of the affine transformations of $\mathbb{R}^{2}$, called quasi-affine transformations (QAT). Then, the study was extended to the $n \mathrm{D}$-spaces [1, 7, 12, 15, 18]. Nevertheless, in these works, the QAT are studied per se and do not result from a discretization scheme, a fortiori not from a multi-scale one. Here, we exhibit the link between an $\Omega$-arithmetization scheme derived from the one of Chollet et al. and sequences of QATs. The arithmetization scheme of Chollet et al. provide a multiscale arithmetic representation of the plane curve $y=x(t)$ solution of the Cauchy problem $x^{\prime}=F(t, x), x\left(t_{0}\right)=x_{0}$. A linear transformation $T$ of the Euclidean plane $\mathbb{R}^{2}$ can be represented by the 2D-plane $\mathbf{y}=T(\mathbf{x})$ in the 4D-space of the pair $(\mathbf{x}, \mathbf{y})$, that is $\mathbb{R}^{2} \times \mathbb{R}^{2}$. What is initiated here is to show that the arithmetization scheme of Chollet et al. can be extended to yield a multiscale arithmetic representation of the plane $\mathbf{y}=T(\mathbf{x})$ as a sequence of $2 \mathrm{D}$ discrete quasi-planes of $\mathbb{Z}^{2} \times \mathbb{Z}^{2}$. We prove the convergence of the scheme toward the Euclidean transformation and we prove that the sequence itself contains the same information as the transformation $T$ since there exists an isomorphism (that we provide) between the Euclidean linear transformations and the quasi-linear integer transformation sequences.

Let us end this introduction by two warnings. Firstly, the reader will not find in the article any solution to the issues raised by the computation of linear transformations, for instance rotations: bijectivity, connectivity and so on. It is just a first step toward a constructive, multi- 
scale, model of such transformations. Secondly, although the tools we use have been designed using non-standard analysis, the presentation we make of them, and our own statements, are within the framework of classical analysis and no knowledge of nonstandard concepts is required to understand the work presented in the remainder of this article.

\section{Background}

\subsection{The Harthong-Reeb line}

The constructive version of the Harthong-Reeb line is quite natural. In short, real numbers are modeled by Cauchy sequences of rationals which in turn are converted to integers thanks to a given sequence of increasing scales. Nevertheless, since a real can be modeled by many rational sequences, it is necessary to use an equivalence relation on such sequences and then to transfer the relation into the integer sequences. Moreover, the addition and the multiplication of the real numbers have to be translated in the language of the integer sequence classes (for the obtained equivalence relation). The most problematic issue is to induce the order relation. In this paper, as we focus on an application to elementary linear algebra, we will not tell anything about the order relation over the HarthongReeb line. The reader who would like a more deep insight into the Harthong-Reeb line should look at [5, Sec. 5].

The two sets, rational sequences and integer sequences, are presented below. A very basic example will end the exposition of the construction. But before, let us give our notations on equivalence relations. In the sequel, given an equivalence relation $\sim$ on a set $X$, we denote by $[a]$ the equivalence class of $a \in X$ and by $\hat{c}$ any representative of the class $c \in X / \sim$ (all the operations defined below are well-defined, that is, they do not depend on the choice of the representatives). Note that the issue of choosing the best representative (in some sense to be precised) is not addressed here. It will be treated in a future work.

Firstly, we present the classes of rational sequences and their operations. Using the Landau notation, we consider the subsets $\mathrm{O}(1)$ and $\mathrm{o}(1)$ of the rational sequences and we denote by $\mathbb{Q}_{\text {lim }}$ the quotient space $\mathrm{O}(1) / \mathrm{o}(1)$ of the bounded sequences of rational up to the sequences converging toward 0 . Then, two sequences that share the same limit are in the same equivalence class and, in a given class, all the sequences converge toward the same limit or no sequence converges.

The two operations of the field $\mathbb{Q}$ extend without difficulty to $\mathbb{Q}_{\text {lim. }}$. For any $(\pi, \rho) \in \mathbb{Q}_{\text {lim }}$, we set:

$$
\begin{aligned}
& \pi+\rho \stackrel{\text { def }}{=}[\hat{\pi}+\hat{\rho}], \\
& \pi \times \rho \stackrel{\text { def }}{=}[\hat{\pi} \times \hat{\rho}],
\end{aligned}
$$

where + and $\times$ are the termwise operations on the rational sequences. In a general way, throughout the article, the operations on the sequences are always performed termwise. The restriction of $\mathbb{Q}_{\lim }$ to the classes of the Cauchy sequences is noted $\mathbb{Q}_{\text {Cau }}$. The limit operator provides an isomorphism from $\left(\mathbb{Q}_{\mathrm{Cau}},+, \times\right)$ to $(\mathbb{R},+, \times)$.

Next, we have to transform rationals into integers. Let $\omega$ be a strictly increasing sequence of positive integers. In the theory of Laugwitz and Schmieden [16], such a sequence is called an infinitely large number. Though our work is presented in a classical way (without any other reference to nonstandard analysis), we keep this denomination which recall from where the framework is originated. So, given the infinitely large number $\omega$, we consider the subsets $\mathrm{O}(\omega)$ and $\mathrm{o}(\omega)$ of $\mathbb{Z}^{\mathbb{N}}$ (Landau notation) and we define the Harthong-Reeb line, $\mathscr{H} \mathscr{R}_{\omega}$, as the quotient space $\mathrm{O}(\omega) / \mathrm{o}(\omega)$. We denote by $={ }_{\omega}$ the corresponding equivalence relation. Then, two integer sequences $u, v$ are equivalent for $=_{\omega}$ iff the rational sequence $u / \omega$ and $v / \omega$ are equivalent $(u / \omega$ and $v / \omega$ are in the same class of $\mathbb{Q}_{\mathrm{lim}}$ ).

The Harthong-Reeb line is equipped with the following operations.

$$
\begin{aligned}
u+v & \stackrel{\text { def }}{=}[\hat{u}+\hat{v}], \\
u \times{ }_{\omega} v & \stackrel{\text { def }}{=}[(\hat{u} \times \hat{v}) \div \omega],
\end{aligned}
$$

where $\div$ denotes the (termwise) integer division: $a \div b=$ $\lfloor a / b\rfloor$. The multiplication is indexed by the corresponding infinitely large number for we will use several of these numbers in the sequel. It is unnecessary for the addition. Note that, in particular, the multiplication is defined to be compatible with the multiplication of $\mathbb{Q}_{\lim }$, that is, forgetting the equivalence classes, $u / \omega \times v / \omega=\left(u \times{ }_{\omega} v\right) / \omega$.

It was proved in [5, 6, 17] that the tuple $\left(\mathscr{H}_{\mathscr{R}},+, \times_{\omega}\right)$ is a commutative ring which is isomorphic to $\mathbb{Q}_{\lim }$ via the 
map $\varphi_{\omega}$ and its converse $\psi_{\omega}$ (both maps are well-defined):

$$
\begin{array}{rr}
\varphi_{\omega}: \mathscr{H} \mathscr{R}_{\omega} \rightarrow \mathbb{Q}_{\lim } & \psi_{\omega}: \mathbb{Q}_{\lim } \rightarrow \mathscr{H} \mathscr{R}_{\omega} \\
\varphi_{\omega}(u)=[\hat{u} / \omega] & \psi_{\omega}(\pi)=[\lfloor\omega \times \hat{\pi}\rfloor]
\end{array}
$$

It should be noted that neither $\mathscr{H} \mathscr{R}_{\omega}$ nor $\mathbb{Q}_{\text {lim }}$ are fields in the classical sense since many of their elements do not have any inverse ${ }^{1}$

We end this section about the Harthong-Reeb line by an example. Let $\omega$ be the sequence $\left\langle 10^{n}\right\rangle$. To obtain the image of $\sqrt{3}$ in $\mathscr{H} \mathscr{R}_{\omega}$, we need a sequence of rationals converging toward $\sqrt{3}$. So, using the Babylonian algorithm, we set $b(0)=3$ and $b(n+1)=(b(n)+$ $3 / b(n)) / 2$ for any $n \in \mathbb{N}$. Then the projection of $b$ on $\mathbb{Q}_{\text {lim }}$ represents the real $\sqrt{3}$ in $\mathbb{Q}_{\text {lim. }}$. Finally, the morphism $\psi_{\omega}$ yields the Harthong-Reeb number which represents $\sqrt{3}$ in $\mathscr{H} \mathscr{R}_{\omega}$. It is the class (for the relation $=\omega)$ of the sequence $\left\langle\left\lfloor 10^{n} b(n)\right\rfloor\right\rangle$, that is the sequence $3,20,175,1732,17320,173205, \ldots$ up to sequences dominated by $10^{n}$ asymptotically.

\section{$2.2 \Omega$-arithmetization scheme}

The $\Omega$-arithmetization scheme provides a way to digitize a real function which is the solution of a Cauchy problem $x^{\prime}(t)=f(t, x(t))$ and $x\left(t_{0}\right)=x_{0}$. The differential equation is solved using the Euler scheme through nonstandard analysis, substituting $\mathscr{H} \mathscr{R}_{\omega}$ to $\mathbb{R}$ and infinitely small steps to standard steps. Then, going back to the very definition of the Harthong-Reeb line and taking any section ${ }^{2}$ of the nonstandard solution, one obtains a sequence of integer functions that converges in some sense toward the real function $x$ that is the solution of the Cauchy problem. The commutative diagram of Fig. 1 summarizes the relation between a real continuous function and the corresponding multi-scale integer function in the $\Omega$-arithmetization scheme as presented in [5].

In the diagram, we use several new notations:

\footnotetext{
${ }^{1}$ In [5] and [17] it is stated that $\forall x \in \mathscr{H} \mathscr{R}_{\omega}, x \neq{ }_{\omega} 0, \exists y \in \mathscr{H} \mathscr{R}_{\omega}$ such that $x \times \omega y=\omega 1$ but the relation $\neq \omega$ is not the negation of the relation $=\omega$ (in [17], the implementation in the Coq system relies on the intuitionistic logic, so it does not make use of the law of the excluded middle). In this paper, the structure $\left(\mathscr{H} \mathscr{R}_{\omega},+, \times_{\omega}\right)$ needs not be a field, just a ring and we make no use of the relation $\neq_{\omega}$ so the assertions can be understood in both classical and intuitionistic logics.

${ }^{2}$ Given an equivalence relation, a section is the choice of a representative while the converse operation is called projection.
}

- $\langle E\rangle$, where $E=\mathbb{R}, \mathbb{Q}$ or $\mathbb{Z}$, is the set of the bounded sequences in $E$;

- $\langle\mathbb{Z}\rangle_{\omega}$ is the set of the integer sequences bounded above by the infinitely large number $\omega$ (up to a constant factor).

- $\left\langle{ }^{n} s\right\rangle$ : sequence whose $n$th term is ${ }^{n} s$;

- i: $x \in \mathbb{R} \mapsto\left\langle{ }^{n} x\right\rangle \in\langle\mathbb{R}\rangle$, where ${ }^{n} x=x$ for any $n$.

Furthermore, to avoid to introduce too much notations for similar notions, we make an abuse of notations by writing $\varphi_{\omega}$ and $\psi_{\omega}$ for functions defined on distinct sets but that perform essentially the same calculations:

$$
\begin{aligned}
& \varphi_{\omega}: t \mapsto t / \omega, \\
& \psi_{\omega}: t \mapsto\lfloor\omega \times t\rfloor,
\end{aligned}
$$

where $t$ is a sequence and the calculation is done termwise, or $t$ is a class of sequences and the calculation is done on each element of the class (then, it is assumed that $\varphi_{\omega}$ or $\psi_{\omega}$ is well defined).

Let us take an example. In order to obtain a multiscale representation of the function $\sqrt{1+x}$ on $[0,1]$, we solve the differential equation

$$
x^{\prime}(t)=\frac{1}{2 x(t)} \text { with } x(0)=1 .
$$

Taking $\beta=\left\langle 2^{n}\right\rangle$ and, as in $[5], \omega=\beta^{2}$, the nonstandard Euler scheme of Chollet et al. yields the sequences of integer values shown on Table 1. Each line of the table gives the arithmetization $\left({ }^{n} f\right)$ of the function $\sqrt{1+t}, t \in[0,1]$ for the pair of scales $\left({ }^{n} \beta,{ }^{n} \omega\right)$. For instance, $\left({ }^{2} f\right)(4)=21$ with scales $\left({ }^{2} \beta,{ }^{2} \omega\right)=(4,16)$. Then, using the functions $\varphi_{(2 \beta)}: t \mapsto t / 4$ and $\left.\varphi_{(2} \omega\right): x \mapsto x / 16$, we obtain a discrete rational approximation of $\sqrt{1+t}, t \in[0,1]$, that is shown in Fig. 2. It was proved in [5, Theorem 1] that the sequence of function $\left\langle{ }^{n} f\right\rangle n$ converges pointwise toward the solution of the Cauchy problem, that is toward the function $\sqrt{1+x}$.

\subsection{Quasi affine transformations}

Let $f: \mathbb{Z}^{d} \rightarrow \mathbb{Z}^{d^{\prime}}$ be an affine transformation $\left(d, d^{\prime} \geq 2\right)$ whose linear part is $f_{0}$. Let $r$ be a positive integer. The $Q A T$ (quasi affine transformation) $\lfloor f\rfloor_{r}$ is defined by

$$
\lfloor f\rfloor_{r}: \mathbb{Z}^{d} \ni \mathbf{p} \mapsto f(\mathbf{p}) \div r \in \mathbb{Z}^{d^{\prime}},
$$




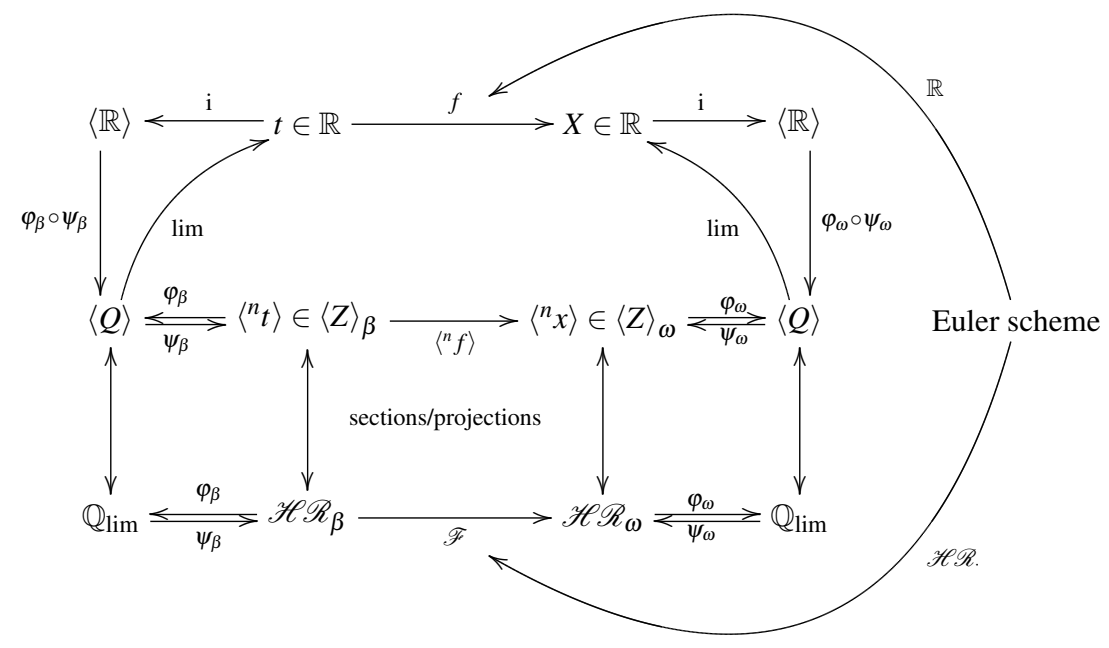

Figure 1: The skeleton of the arithmetization scheme described in [5] which links the Euclidean function $f$ with a sequence of integer functions $\left\langle{ }^{n} f\right\rangle$. Basically, the integer function ${ }^{n} f$ is the arithmetized expression at the scale $n$ of the Euclidean function $f$. The sequence $\left\langle{ }^{n} f\right\rangle$ itself is obtained as a section of the function $\mathscr{F}$ which translate $f$ when $\mathbb{R}$ is replaced by the Harthong-Reeb line. The function $\mathscr{F}$ is computed using a nonstandard version of the Euler scheme for solving differential equations.

\begin{tabular}{l|lllllllll}
\hline & & & & & & & & & \\
$n \backslash t$ & 0 & 1 & 2 & 3 & 4 & 5 & 6 & 7 & 8 \\
\hline 0 & 1 & 1 & & & & & & & \\
1 & 4 & 5 & 5 & & & & & & \\
2 & 16 & 18 & 19 & 20 & 21 & & & & \\
3 & 64 & 68 & 71 & 74 & 77 & 80 & 83 & 86 & 88 \\
4 & 256 & 264 & 271 & 278 & 285 & 292 & 299 & 305 & 311 \\
\hline
\end{tabular}

Table 1: The function $t \mapsto \sqrt{1+t}, t \in[0,1]$, represented as a function from $\mathscr{H}_{\mathscr{R}_{\left\langle 2^{n}\right\rangle}}$ to $\mathscr{H}_{\left\langle 4^{n}\right\rangle}$ (see text).

where $\div$ stands for the integer division : $p \div q=\lfloor p / q\rfloor$.

A $Q L T$ (quasi linear transformation) is a QAT that leaves invariant the origin: $\lfloor f\rfloor_{r}$ is a QLT if $f(\mathbf{p})=$ $f_{0}(\mathbf{p})+\mathbf{q}$ where $0 \leq \mathbf{q}<r$ coordinatewise. In practice, the parameter $\mathbf{q}$ is used to set the rounding mode of the computation.

Given a QLT $g$, the triple $\left(r, f_{0}, \mathbf{q}\right)$ defining $g$ that has the smallest $r$ is called the canonical representative of $g$ and $r$ is called the scale of $g$. The QLTs model the Euclidean linear transformations when they are carried out

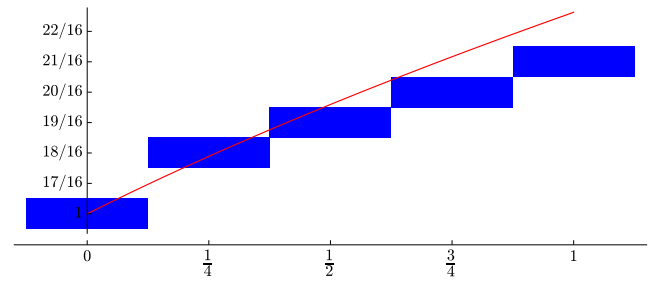

Figure 2: The discretization of the curve $y=\sqrt{1+x}$ at the scale $(4,16)$ provided by the $\Omega$-arithmetization scheme.

by a computer on a discrete grid. For instance, a rotation about the origin through an angle of $\pi / 3$ could be modeled by the QLT whose canonical representative is $\left(100,\left(\begin{array}{cc}50 & -173 \\ 173 & 50\end{array}\right), 50\right)$.

The reader interested by the properties of QATs and QLTs is referred back to the articles cited in the introduction.

In the following section, we show that the $\Omega$-arithmetization scheme extends well to linear transformations between Euclidean spaces, involving sequences of QLTs. 


\section{$3 \Omega$-arithmetization of affine trans- formations}

Let us consider again the commutative diagram presented in Fig. 1. In this section, we want to generalize it by replacing the Euclidean function $f$ by a linear transformation between $\mathbb{R}^{d}$ and $\mathbb{R}^{d^{\prime}}$. Since affine transformations are easily characterized by their matrices, we don't need to use any numerical algorithm like the Euler scheme. Generally, when computing a linear transformation of a picture, one tunes the calculus precision independently of the space resolution. So, we need two infinitely large numbers, denoted by $\alpha$ and $\omega$. Practically, for a linear transformation over $\mathbb{R}^{d}$, the sequence $\alpha$ can be viewed as a sequence of resolutions - or a sequence of finer and finer grids-while the sequence $\omega$ allows to quantify the matrix coefficients more and more accurately. Nevertheless, multiplying integers modeling real numbers with distinct scales requires to specify the scale of the result. Technically, $\alpha \neq \omega$ implies generally that the rings $\mathscr{H} \mathscr{R}_{\alpha}$ and $\mathscr{H} \mathscr{R}_{\omega}$ are distinct. Thus, we need to extend the inner product of $\mathscr{H} \mathscr{R}_{\omega}$ to an outer product over $\mathscr{H} \mathscr{R}_{\omega} \times \mathscr{H} \mathscr{R}_{\alpha}$ with values in a third ring $\mathscr{H} \mathscr{R}_{\beta}$. It is natural to choose $\beta$ as the geometric mean of $\alpha$ and $\omega: \beta^{2}=\alpha \times \omega$. This is confirmed by the following property which expresses that, when a pair in $\mathscr{H} \mathscr{R}_{\omega} \times \mathscr{H} \mathscr{R}_{\alpha}$ is sent in $\mathscr{H} \mathscr{R}_{\beta}$ via the maps $\varphi$. and $\psi$., the product is expressed as the inner product of $\mathscr{H} \mathscr{R}_{\beta}$.

Lemma 1. Let $\alpha, \beta$, $\omega$ be three infinitely large numbers such that $\beta^{2}$ is equal to $\alpha \times \omega$ asymptotically: $\beta^{2} \sim \alpha \times$ $\omega$. Then, for any $u \in \mathscr{H} \mathscr{R}_{\alpha}, v \in \mathscr{H} \mathscr{R}_{\omega}$,

$$
\psi_{\beta} \circ \varphi_{\omega}(v) \times_{\beta} \psi_{\beta} \circ \varphi_{\alpha}(u)=[(\hat{u} \times \hat{v}) \div \beta] .
$$

Proof. Let $A$ be a representative of $\psi_{\beta} \circ \varphi_{\omega}(v) \times_{\beta} \psi_{\beta}$ ○ $\varphi_{\alpha}(u)$. Going back to the very definitions of $\varphi, \psi$ and $\times_{\beta}$, we have

$$
\begin{aligned}
{[A] } & =\left[\left\lfloor\beta \frac{\hat{v}}{\omega}\right\rfloor\right] \times \times_{\beta}\left[\left\lfloor\beta \frac{\hat{u}}{\alpha}\right\rfloor\right] \text { and then, } \\
A & =\beta\left(\left\lfloor\beta \frac{\hat{v}}{\omega}\right\rfloor \times\left\lfloor\beta \frac{\hat{u}}{\alpha}\right\rfloor\right) \div \beta .
\end{aligned}
$$

Then, writing $\lfloor\beta \hat{v} / \omega\rfloor$ and $\lfloor\beta \hat{u} / \omega\rfloor$ as $\beta \hat{v} / \omega+\mathrm{O}(1)$ and $\beta \hat{u} / \omega+\mathrm{O}(1)$, we get

$$
A=\beta\left(\frac{\beta^{2}}{\alpha \omega} \hat{v} \hat{u}+\mathrm{O}\left(\beta \frac{\hat{v}}{\omega}\right)+\mathrm{O}\left(\beta \frac{\hat{u}}{\alpha}\right)\right) \div \beta .
$$

Since, by hypothesis, $\beta^{2} / \alpha \omega=1+\mathrm{o}(1), \hat{v} \in \mathrm{O}(\omega), \hat{u} \in$ $\mathrm{O}(\alpha)$, we derive

$$
A=\beta(\hat{v} \hat{u}+\mathrm{o}(\hat{v} \hat{u})+\mathrm{O}(\beta)) \div \beta
$$

Invoking again the same hypotheses, we obtain

$$
A=\beta(\hat{v} \hat{u}) \div \beta+\mathrm{o}(\beta)+\mathrm{O}(1)
$$

Because $\beta$ is an infinitely large number and by definition of the relation $=\beta$ the latter equation implies

$$
[A]=(\hat{v} \hat{u}) \div \beta
$$

Then, given two infinitely large numbers $\alpha, \omega$ whose geometric mean is asymptotically equal to the infinitely large number $\beta$, we define the outer product $\times_{\alpha}^{\omega}$ as follows:

$$
\begin{aligned}
\mathscr{H} \mathscr{R}_{\omega} \times \mathscr{H} \mathscr{R}_{\alpha} \longrightarrow \mathscr{H} \mathscr{R}_{\beta} \\
(v, u) \longmapsto v \times{ }_{\alpha}^{\omega} u \stackrel{\text { def }}{=}[(\hat{u} \times \hat{v}) \div \beta] .
\end{aligned}
$$

From Lemma 1, because the maps $\psi$ and $\varphi$ are morphisms, we readily derive that this product is well-defined and satisfies the following two properties which ensure the linearity of the product:

$$
\begin{aligned}
& \forall u_{1}, u_{2} \in \mathscr{H} \mathscr{R}_{\alpha}, \forall v \in \mathscr{H} \mathscr{R}_{\omega}, \\
& v \times_{\alpha}^{\omega}\left(u_{1}+u_{2}\right)=v \times_{\alpha}^{\omega} u_{1}+v \times_{\alpha}^{\omega} u_{2} ; \\
& \forall \lambda, u \in \mathscr{H} \mathscr{R}_{\alpha}, \forall v \in \mathscr{H} \mathscr{R}_{\omega}, \\
& v \times_{\alpha}^{\omega}(\lambda u)=\psi_{\beta} \circ \varphi_{\alpha}(\lambda)\left(v \times_{\alpha}^{\omega} u\right) .
\end{aligned}
$$

Provided $\alpha \times \omega \sim \beta^{2}$, the outer product $\times_{\alpha}^{\omega}$ allows to define a matrix product from $\mathscr{M}_{d^{\prime} d}\left(\mathscr{H} \mathscr{R}_{\omega}\right) \times \mathscr{M}_{d, 1}\left(\mathscr{H} \mathscr{R}_{\alpha}\right)$ to $\mathscr{M}_{d^{\prime}, 1}\left(\mathscr{H} \mathscr{R}_{\beta}\right)$. Note that this matrix product is a shortcut for a succession of morphisms. Indeed, thanks to the pair of converse isomorphisms $(\varphi, \psi)$, the HarthongReeb lines $\mathscr{H} \mathscr{R}_{\alpha}$ and $\mathscr{H} \mathscr{R}_{\beta}$ are bound to $\mathscr{H} \mathscr{R}_{\omega}$ via the 
following isomorphisms:

$$
\begin{aligned}
\psi_{\omega} \circ \varphi_{\alpha}: \mathscr{H} \mathscr{R}_{\alpha} & \longrightarrow \mathscr{H} \mathscr{R}_{\omega} \\
x & \longmapsto\left[\left\lfloor\frac{\hat{x}}{\alpha}\right\rfloor\right] \\
\psi_{\omega} \circ \varphi_{\beta}: \mathscr{H} \mathscr{R}_{\beta} & \longrightarrow \mathscr{H}_{\mathscr{R}_{\omega}} \\
x & \longmapsto\left[\left\lfloor\omega \frac{\hat{x}}{\beta}\right\rfloor\right] .
\end{aligned}
$$

Then, using the outer matrix product is equivalent to applying $\psi_{\omega} \circ \varphi_{\alpha}$ on the vector coordinates, then to perform the inner matrix product and finally to apply $\psi_{\beta} \circ \varphi_{\omega}$ on the resulting coordinates.

Eventually, the proposed arithmetization scheme for the Euclidean linear transformations is defined in the commutative diagram of Fig. 4. In the scheme, the starting point is the $d^{\prime} \times d$ matrix $\mathrm{F}$ with real coefficients. These coefficients yield Harthong-Reeb versions in $\mathscr{H} \mathscr{R}_{\omega}$ which results in a matrix $L$ and a map $\ell$ between the modules $\mathscr{H} \mathscr{R}_{\alpha}{ }^{d}$ and $\mathscr{H} \mathscr{R}_{\beta}{ }^{d^{\prime}}$. We say that $\ell$ is an $\Omega$ linear transformation $(\Omega$-LT) derived form the matrix $L$. In the following section, we study the relation between the map $\ell$ and the Euclidean linear map $f$.

\section{$3.1 \Omega$-LT}

In the commutative diagramme of Fig 3 , following the external arrows from $\mathbb{R}^{e}$ to $\mathscr{H} \mathscr{R}_{\gamma}{ }^{e}$, where $(e, \gamma) \in$ $\left\{(d, \alpha),\left(d^{\prime}, \beta\right)\right\}$, we observe that, for any real vector $\mathbf{x}$,

$$
\begin{aligned}
\psi_{\gamma}\left(\left[\varphi_{\gamma} \circ \psi_{\gamma}(\mathrm{i}(\mathbf{x}))\right]\right) & =\left[\psi_{\gamma} \circ \varphi_{\gamma} \circ \psi_{\gamma}(\mathrm{i}(\mathbf{x}))\right] \\
& =\left[\psi_{\gamma}(\mathrm{i}(\mathbf{x}))\right] \\
& =[\lfloor\gamma \mathbf{x}\rfloor] .
\end{aligned}
$$

The second equality is true because $\psi_{\gamma} \circ \varphi_{\gamma}$ is the floor function and $\psi_{\gamma}(\mathrm{i}(\mathbf{x}))$ is an integer. Note that we still make an abuse of notation. Indeed, $\mathbf{x}$ is a vector and the calculus must be understood as a parallel calculus on each coordinate of $\mathbf{x}$. Now, we denote by $\xi_{\gamma}^{\mathrm{HR}}$ the function from $\mathbb{R}^{e}$ to $\mathscr{H} \mathscr{R}_{\gamma}{ }^{e}$ that maps $\mathbf{x}$ to $[\lfloor\gamma \mathbf{x}\rfloor]$. This function is a ring morphism since the maps i, $\varphi, \psi$ and the projection map of the equivalence relation are all ring morphisms. Furthermore, $\xi_{\gamma}^{\mathrm{HR}}$ is an injection. Indeed, $\lfloor\gamma \mathbf{x}\rfloor=\gamma\lfloor\gamma \mathbf{y}\rfloor$ implies $\gamma(\mathbf{x}-\mathbf{y}) \in \mathrm{o}(\gamma)$ and then $\mathbf{x}=\mathbf{y}$ since $\mathbf{x}$ and $\mathbf{y}$ are constant vectors. The converse function, defined on $\xi_{\gamma}^{\mathrm{HR}}\left(\mathbb{R}^{e}\right)$, maps a vector $\mathbf{u} \in \xi_{\gamma}^{\mathrm{HR}}\left(\mathbb{R}^{e}\right)$ to the real vector $\lim \hat{\rho}$ where $\rho=\varphi_{\gamma}(\mathbf{u})$. Indeed, assume $\mathbf{u}=\xi_{\gamma}^{\mathrm{HR}}(\mathbf{x})$, that is, $\mathbf{u}=[\lfloor\gamma \mathbf{x}\rfloor]$. Then, by definition of $\varphi_{\gamma}$, $\hat{\rho}=\lfloor\gamma \mathbf{x}\rfloor / \gamma$ and the sequence $\lfloor\gamma \mathbf{x}\rfloor / \gamma$ converge toward $x$. Note that the choice of the representative $\hat{\rho}$ does not matter since, by definition of $\mathbb{Q}_{\text {lim }}$, all the sequences belonging to the class $\rho$ share the same limit if any. The converse map can be extended to $\mathscr{H} \mathscr{R}_{\gamma}{ }^{e}$ by the function $\chi_{\gamma}^{\mathrm{HR}}: \mathscr{H}_{\mathscr{R}}{ }^{e} \rightarrow \mathbb{R}^{e}$ defined by $\chi_{\gamma}^{\mathrm{HR}}(\mathbf{u})=\lim \sup (\hat{\rho})$ where $\rho=\varphi_{\gamma}(\mathbf{u})$. Again, we observe that the choice of the representative $\hat{\rho}$ does not matter since two sequences whose difference tends toward zero have the same upper limit. We have $\chi_{\gamma}^{\mathrm{HR}} \circ \xi_{\gamma}^{\mathrm{HR}}=$ id where id is the identity map. The converse map $\xi_{\gamma}^{\mathrm{HR}} \circ \chi_{\gamma}^{\mathrm{HR}}$ is neither injective nor surjective. Nevertheless, its restriction to $\xi_{\gamma}^{\mathrm{HR}}\left(\mathbb{R}^{d}\right)$ is the identity map.

We are now able to specify the relation between the linear map $\ell$ and the $\Omega$-LT $\ell$ in the commutative diagram of Fig 3

Proposition 1. Let $f$ be a linear transformation between $\mathbb{R}^{d}$ and $\mathbb{R}^{d^{\prime}}$ whose matrix is $F=\left(F_{i, j}\right)$ and $\alpha, \beta$ be two infinitely large numbers such that $\alpha \in o\left(\beta^{2}\right)$. Then, setting $\omega=\beta^{2} \div \alpha$, the $\Omega$-LT $\ell$ from $\mathscr{H} \mathscr{R}_{\alpha}{ }^{d}$ to $\mathscr{H} \mathscr{R}_{\beta}{ }^{d^{\prime}}$ whose matrix is $L=\left(\xi_{\omega}^{\mathrm{HR}}\left(F_{i, j}\right)\right)$ is such that

$$
\xi_{\beta}^{\mathrm{HR}} \circ f=\ell \circ \xi_{\alpha}^{\mathrm{HR}} .
$$

In other words, one has the commutative diagram:

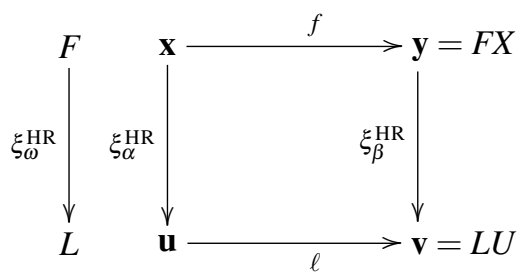

Proof. Firstly, we observe that, for any infinitely large number $\gamma$, one has

$$
\xi_{\beta}=\psi_{\beta} \circ \varphi_{\gamma} \circ \xi_{\gamma}
$$

because $\lfloor\beta\lfloor\omega x\rfloor / \omega\rfloor={ }_{\beta}\lfloor\beta x\rfloor$ for any $x \in \mathbb{R}$. Then, since $\xi_{\beta}^{\mathrm{HR}}$ is a ring morphism,

$$
\begin{aligned}
\xi_{\beta}^{\mathrm{HR}}(\mathrm{Fx}) & =\left(\xi_{\beta}^{\mathrm{HR}}\left(\sum \mathrm{F}_{i, j} \times \mathrm{X}_{j}\right)\right)_{j} \\
& =\left(\sum \xi_{\beta}^{\mathrm{HR}}\left(\mathrm{F}_{i, j}\right) \times_{\beta} \xi_{\beta}^{\mathrm{HR}}\left(\mathrm{X}_{j}\right)\right)_{j}
\end{aligned}
$$




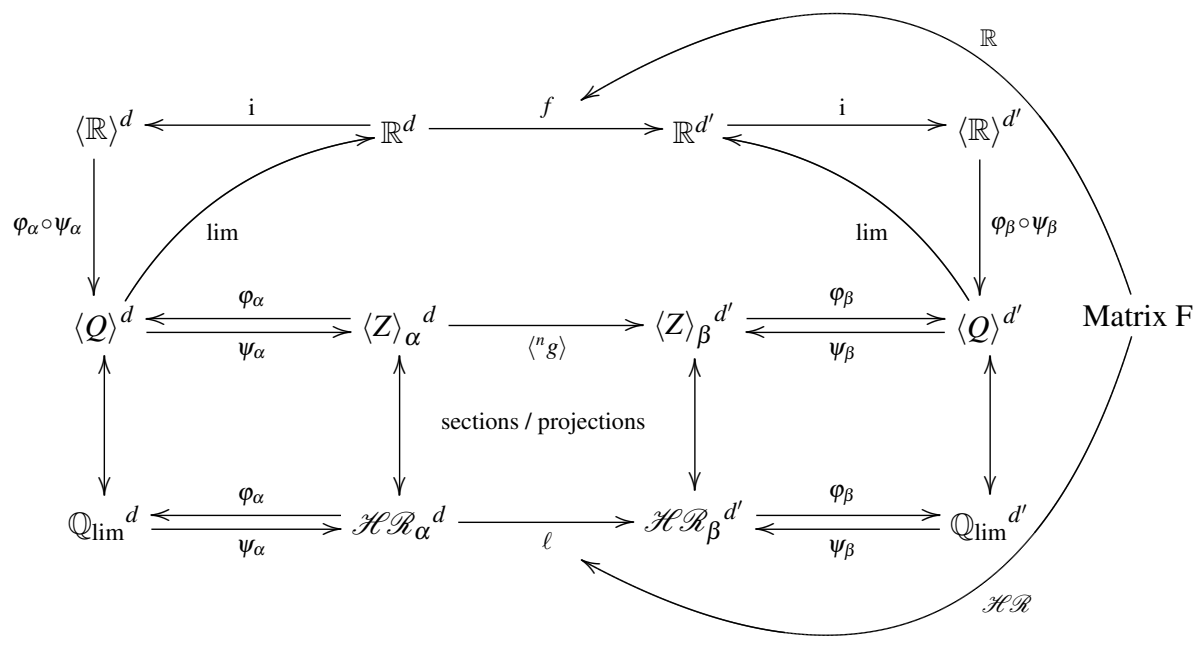

Figure 3: The $\Omega$-arithmetization scheme which associates to the linear transformation $f$ the sequence of quasi-affine transformations $\left\langle{ }^{n} g\right\rangle$ from $\langle\mathbb{Z}\rangle_{\alpha}{ }^{d}$ to $\langle\mathbb{Z}\rangle_{\beta}{ }^{d^{\prime}}$. This sequence is obtained as a section of a translation of the matrix $\mathrm{F}$ of $f$ using Harthong-Reeb coefficients.

Thus, using (1),

$$
\begin{aligned}
& \xi_{\beta}^{\mathrm{HR}}(\mathrm{F} \mathbf{x})= \\
& \left(\sum \psi_{\beta} \circ \varphi_{\omega}\left(\xi_{\omega}^{\mathrm{HR}}\left(\mathrm{F}_{i, j}\right)\right) \times_{\beta} \psi_{\beta} \circ \varphi_{\alpha}\left(\xi_{\alpha}^{\mathrm{HR}}\left(\mathrm{X}_{j}\right)\right)\right)_{j} .
\end{aligned}
$$

Then, from Lemma11.

$$
\begin{aligned}
\xi_{\beta}^{\mathrm{HR}}(\mathrm{F} \mathbf{x}) & =\left(\sum \xi_{\omega}^{\mathrm{HR}}\left(\mathrm{F}_{i, j}\right) \times_{\alpha}^{\omega} \xi_{\alpha}^{\mathrm{HR}}\left(\mathrm{X}_{j}\right)\right)_{j} \\
& =L \mathbf{u} .
\end{aligned}
$$

Prop. 1 enables to compute the linear map $f$ from the $\Omega$-LT $\ell$ and, conversely, to compute $\ell$ from $f$ but only for the vectors of $\mathscr{H} \mathscr{R}_{\alpha}$ that lie in $\xi_{\alpha}^{\mathrm{HR}}\left(\mathbb{R}^{d}\right)$. Note that we are mostly interested in the former computation which allow us to compute a Euclidean linear transformation at given scales through integer only computations.

Corollary 1. With the notations of Prop. 1] one has

$$
\begin{aligned}
f & =\chi_{\beta} \circ \ell \circ \xi_{\alpha}^{\mathrm{HR}} ; \\
\ell_{\uparrow \xi_{\alpha}^{\mathrm{HR}}\left(\mathbb{R}^{d}\right)} & =\xi_{\beta}^{\mathrm{HR}} \circ f \circ \chi_{\alpha} .
\end{aligned}
$$

Before giving the proof, observe that, since the map $\chi_{\beta}$ is essentially a limit operator, Eq. (2) is a pointwise convergence property of some sequences of maps from $\mathbb{R}^{d}$ to $\mathbb{Q}^{d^{\prime}}$ toward the linear map $f$. It is the object of Sec. 3.2 to describe more precisely these maps while Sec. 3.3 study more precisely the convergence.

Proof. (of Corollary 1)

In the diagram of Prop. 1 , we have $\mathbf{u} \in \xi_{\alpha}^{\mathrm{HR}}\left(\mathbb{R}^{d}\right)$ and $\mathbf{v} \in$ $\xi_{\beta}^{\mathrm{HR}}\left(\mathbb{R}^{d^{\prime}}\right)$. Since the restriction of $\chi_{\alpha}$ to $\xi_{\alpha}^{\mathrm{HR}}\left(\mathbb{R}^{d}\right)$ is the inverse function of $\xi_{\alpha}^{\mathrm{HR}}$ and, alike, the restriction of $\chi_{\beta}$ to $\xi_{\beta}^{\mathrm{HR}}\left(\mathbb{R}^{d^{\prime}}\right)$ is the converse function of $\xi_{\beta}^{\mathrm{HR}}$, we conclude straightforwardly.

In the following section we study the relation between the map $\ell$ and the sequence of maps $\left\langle{ }^{n} g\right\rangle$ in the diagram of Fig. 3 We will show that the maps ${ }^{n} g$ are QLTs.

\section{$3.2 \Omega$-LTs and QATs}

Tentatively, forgetting the scheme illustrated in Fig. 3, we now focus on the understanding of the $\Omega$-LTs per se, that is going back to the very definition of $\mathscr{H} \mathscr{R}_{\omega}$. 
Firstly, we show that the definition of the multiplication in $\mathscr{H} \mathscr{R}_{\omega}$ has a natural extension with matrices.

Lemma 2. Let $\alpha, \beta$, $\omega$ be three infinitely large numbers such that $\alpha \omega \sim \beta^{2}$. Let $W \in \mathscr{M}_{d^{\prime}, d}\left(\mathscr{H} \mathscr{R}_{\omega}\right)$ be a matrix and $U \in \mathscr{M}_{d, 1}\left(\mathscr{H} \mathscr{R}_{\alpha}\right)$ be a column vector. Then,

$$
W U=[(\widehat{W} \widehat{U}) \div \beta]
$$

where $\widehat{W}$ is any matrix $\left(\widehat{W}_{i, j}\right)_{i, j}, \widehat{U}$ is any vector $\left(\widehat{U}_{j}\right)_{j}$.

Proof. Starting from the matrix product definition, we have

$$
\begin{aligned}
\mathrm{WU} & =\left(\sum_{j} \mathrm{~W}_{i, j} \times{ }_{\alpha}^{\omega} \mathrm{U}_{j}\right)_{i} \\
& =\left(\sum_{j}\left[\left(\widehat{\mathrm{W}}_{i, j} \times \widehat{\mathrm{U}}_{j}\right) \div \beta\right]\right)_{i},
\end{aligned}
$$

and, since + is compatible with $=_{\beta}$,

$$
\begin{aligned}
\mathrm{WU} & =\left(\left[\sum_{j}\left(\widehat{\mathrm{W}}_{i, j} \times \widehat{\mathrm{U}}_{j}\right) \div \beta\right]\right)_{i} \\
& =[(\widehat{\mathrm{W}} \widehat{\mathrm{U}}) \div \beta] .
\end{aligned}
$$

It appears that Lemma2 is the key to introduce the relation between $\Omega$-LTs defined over Harthong-Reeb spaces and quasi-linear transformations over integer spaces. Let $\ell: \quad \mathscr{H} \mathscr{R}_{\alpha}{ }^{d} \rightarrow \mathscr{H} \mathscr{R}_{\beta}{ }^{d^{\prime}}$ be an $\Omega$-LT whose matrix is noted $L\left(L \in \mathscr{M}_{d^{\prime}, d}\left(\mathscr{H} \mathscr{R}_{\omega}\right)\right.$ where $\left.\omega=\beta^{2} \div \alpha\right)$. Let $\widehat{L}=\left\langle{ }^{n} L\right\rangle$ be a sequence of integer matrices such that $\left[\left\langle{ }^{n} L_{i, j}\right\rangle\right]=L_{i, j}$. We write $\hat{\ell}$, or $\left\langle{ }^{n} \ell\right\rangle$, for the sequence of QLTs whose canonical representatives are $\left({ }^{n} \beta,{ }^{n} L, \mathbf{0}\right)$ and, given a sequence $\mathbf{p}=\left\langle{ }^{n} \mathbf{p}\right\rangle$ of integer vectors, we denote by $\hat{\ell}(\mathbf{p})$ the sequence $\left\langle{ }^{n} \ell\left({ }^{n} \mathbf{p}\right)\right\rangle$.

Let us illustrate these notations with an example. We consider the matrix $\mathrm{F}$ of the rotation through an angle of $\pi / 3$ :

$$
F=\left(\begin{array}{cc}
1 / 2 & -\sqrt{3} / 2 \\
\sqrt{3} / 2 & 1 / 2
\end{array}\right)
$$

and we assume $\alpha=\left\langle 2^{n}\right\rangle$ and $\omega=\left\langle 10^{n}\right\rangle$. Then, for $\beta$, we take the geometric mean of $\alpha$ and $\omega$ :

$$
\beta=\left\langle\left\lfloor\sqrt{20^{n}}\right\rfloor\right\rangle
$$

The corresponding matrix in $\mathscr{M}_{d^{\prime} d}\left(\mathscr{H} \mathscr{R}_{\omega}\right)$ is $L=$ $\left(\begin{array}{ll}L_{1,1} & L_{1,2} \\ L_{2,1} & L_{2,2}\end{array}\right)$ where

$$
\begin{aligned}
& L_{1,1}=L_{2,2}=[\langle 0,5,50,500, \ldots\rangle] \text { and, } \\
& L_{2,1}=-L_{1,2}=[\langle 0,8,86,866, \ldots\rangle] .
\end{aligned}
$$

Then, the sequence of matrices $\widehat{L}=\left\langle{ }^{n} L\right\rangle$ can be set as

$$
\begin{aligned}
{ }^{0} L & =\left(\begin{array}{ll}
0 & 0 \\
0 & 0
\end{array}\right), & { }^{1} L & =\left(\begin{array}{cc}
5 & -8 \\
8 & 5
\end{array}\right), \\
{ }^{2} L & =\left(\begin{array}{cc}
50 & -86 \\
86 & 50
\end{array}\right), & { }^{3} L & =\left(\begin{array}{cc}
500 & -866 \\
866 & 500
\end{array}\right),
\end{aligned}
$$

Thereby, the sequence of QLTs $\hat{\ell}$ is defined by

$$
\begin{array}{ll}
\mathrm{P} \stackrel{{ }^{0} \ell}{\longmapsto}\left\lfloor\left(\begin{array}{ll}
0 & 0 \\
0 & 0
\end{array}\right) \mathrm{P}\right\rfloor, \quad & \mathrm{P} \stackrel{{ }^{1} \ell}{\longmapsto}\left\lfloor\frac{1}{4}\left(\begin{array}{cc}
5 & -8 \\
8 & 5
\end{array}\right) \mathrm{P}\right\rfloor, \\
\mathrm{P} \stackrel{{ }^{2} \ell}{\longmapsto}\left\lfloor\frac{1}{20}\left(\begin{array}{cc}
50 & -86 \\
86 & 50
\end{array}\right) \mathrm{P}\right\rfloor, & \mathrm{P} \stackrel{{ }^{3} \ell}{\longmapsto}\left\lfloor\frac{1}{89}\left(\begin{array}{cc}
500 & -866 \\
866 & 500
\end{array}\right) \mathrm{P}\right\rfloor, \\
\ldots &
\end{array}
$$

Taking $\mathbf{p}=\langle(0,0),(1,1),(2,2),(5,5), \ldots\rangle$, whose equivalence class represents the vector $\exp (\mathrm{i} \pi / 4)$ in $\mathscr{H} \mathscr{R}_{\alpha}$, the sequence of vectors $\widehat{L}(\mathbf{p})$ is

$$
\langle(0,0),(-1,3),(-3,13),(-21,76), \ldots\rangle
$$

(to be compare with the vector $\exp (\mathrm{i}(7 \pi / 12))$ at the scales yielded by $\beta$ ).

Then, from Lemma 2, we derive the next assertion that links $\Omega$-LTs and the related sequences of QLTs, that is, basically, matrices of sequences and sequences of matrices.

Corollary 2. Let $\ell: \mathscr{H} \mathscr{R}_{\alpha}{ }^{d} \rightarrow \mathscr{H} \mathscr{R}_{\beta}{ }^{d^{\prime}}$ be an $\Omega-L T$. Then, for any vector $\mathbf{u} \in \mathscr{H} \mathscr{R}_{\alpha}{ }^{d}$, one has

$$
\ell(\mathbf{u})=[\hat{\ell}(\hat{\mathbf{u}})] .
$$

Proof. Let $L$ and $U$ be the matrices arising from $\ell$ and $\mathbf{u}$.

On the one hand, the $n$th element of the sequence $\hat{\ell}(\hat{\mathbf{u}})$ is the vector ${ }^{n} \ell\left({ }^{n} \hat{\mathbf{u}}\right)$ which, by definition of ${ }^{n} \ell$, has matrix $\left({ }^{n} L^{n} \widehat{\mathrm{U}}\right) \div{ }^{n} \beta$.

On the other hand, from Lemma 2, the vector $\ell(\mathbf{u})$ has matrix $[(\widehat{L} \widehat{U}) \div \beta]$ and, since,$+ \times$ and $\div$ are termwise operations in $\mathbb{Z}^{\mathbb{N}}$, the $n$th element of the sequence $(\widehat{L} \widehat{\mathrm{U}}) \div$ $\beta$ is $\left({ }^{n} L^{n} \hat{\mathbf{u}}\right) \div{ }^{n} \beta$. 
The following theorem characterizes the relationship between the $\Omega$-LT from $\mathscr{H} \mathscr{R}_{\alpha}^{d}$ to $\mathscr{H} \mathscr{R}_{\beta}^{d^{\prime}}$ and the quasilinear transformations from $\mathbb{Z}^{d}$ to $\mathbb{Z}^{d^{\prime}}$.

Theorem 1. Let $\ell$ be a map from $\mathscr{H} \mathscr{R}_{\alpha}{ }^{d}$ to $\mathscr{H} \mathscr{R}_{\beta}{ }^{d^{\prime}}$ where $\alpha \in o\left(\beta^{2}\right)$. Let $\omega=\beta^{2} \div \alpha$.

If $\ell$ is an $\Omega-L T$ with matrix $L$, then, for any sequence $\left\langle{ }^{n} g\right\rangle$ of QLTs with canonical representatives $\left({ }^{n} \gamma,{ }^{n} G,{ }^{n} \mathbf{p}\right)$ such that $\left\langle{ }^{n} \gamma\right\rangle={ }_{\beta} \beta,\left\langle\left\|{ }^{n} G\right\|_{\infty}\right\rangle \in O(\omega)$ and $\left[\left\langle{ }^{n} G\right\rangle\right]=L$, one has, for any $\mathbf{u} \in \mathscr{H} \mathscr{R}_{\alpha}{ }^{d}, \ell(\mathbf{u})=\left[\left\langle{ }^{n} g\left({ }^{n} \hat{\mathbf{u}}\right)\right\rangle\right]$.

Conversely, $\ell$ is an $\Omega-L T$ if there exists a sequence of $Q L T s\left\langle{ }^{n} g\right\rangle$ with scales ${ }^{n} \gamma$ such that $\left\langle{ }^{n} \gamma\right\rangle={ }_{\omega} \beta,\left\langle\left\|{ }^{n} G\right\|_{\infty}\right\rangle \in$ $O(\omega)$ and, for any $\mathbf{u} \in \mathscr{H} \mathscr{R}_{\alpha}{ }^{d}$, there exists $\hat{\mathbf{u}}$ such that $\ell(\mathbf{u})=\left[\left\langle{ }^{n} g\left({ }^{n} \hat{\mathbf{u}}\right)\right\rangle\right]$.

Proof. Firstly, we assume that $\ell$ is an $\Omega$-LT.

Let $L \in \mathscr{M}_{d, d^{\prime}}\left(\mathscr{H} \mathscr{R}_{\omega}\right)$ be the matrix of $\ell$. Let $\left\langle{ }^{n} g\right\rangle$ be a sequence of QLTs whose canonical representatives are are the triples $\left({ }^{n} \gamma,{ }^{n} \mathrm{G},{ }^{n} \mathbf{p}\right)$ where $\left\langle{ }^{n} \gamma\right\rangle={ }_{\beta} \beta$ and $\left[\left\langle{ }^{n} \mathrm{G}\right\rangle\right]=$ $L$. We derive from Corollary 2 that, for any vector $\mathbf{u} \in \mathscr{H} \mathscr{R}_{\alpha}{ }^{d}, \ell(\mathbf{u})$ has a representative whose $n$th term is $\left({ }^{n} \mathrm{G}^{n} \hat{\mathbf{u}}\right) \div{ }^{n} \beta$. Then, to achieve the first part of the proof, it suffices to show that the sequences $\left\langle\left({ }^{n} \mathrm{G}^{n} \hat{\mathbf{u}}\right) \div{ }^{n} \beta\right\rangle$ and $\left\langle{ }^{n} g\left({ }^{n} \hat{\mathbf{u}}\right)\right\rangle$ are equivalent for the relation $=\beta$.

$$
\begin{aligned}
\left|\left({ }^{n} \mathrm{G}\left({ }^{n} \hat{\mathbf{u}}\right) \div{ }^{n} \beta\right)-{ }^{n} g\left({ }^{n} \hat{\mathbf{u}}\right)\right| \\
\quad=\left|\left({ }^{n} \mathrm{G}\left({ }^{n} \hat{\mathbf{u}}\right) \div{ }^{n} \beta\right)-\left(\left({ }^{n} \mathrm{G}\left({ }^{n} \hat{\mathbf{u}}\right)+{ }^{n} \mathbf{p}\right) \div{ }^{n} \gamma\right)\right| \\
\leq 1+\left|\frac{{ }^{n} \mathrm{G}\left({ }^{n} \hat{\mathbf{u}}\right)}{{ }^{n} \beta}-\frac{{ }^{n} \mathrm{G}\left({ }^{n} \hat{\mathbf{u}}\right)+{ }^{n} \mathbf{p}}{{ }^{n} \gamma}\right| \\
\quad \leq 1+\left|\frac{{ }^{n} \mathrm{G}\left({ }^{n} \hat{\mathbf{u}}\right)}{{ }^{n} \beta}\right|\left|1-\frac{{ }^{n} \beta}{{ }^{n} \gamma}\right|+\left|\frac{{ }^{n} \mathbf{p}}{{ }^{n} \gamma}\right| .
\end{aligned}
$$

Since, for any integer $n,{ }^{n} g$ is a QLT, we derive that

$$
\left|\left({ }^{n} \mathrm{G}\left({ }^{n} \hat{\mathbf{u}}\right) \div{ }^{n} \beta\right)-{ }^{n} g\left({ }^{n} \hat{\mathbf{u}}\right)\right| \leq 2+\left|\frac{{ }^{n}\left({ }^{n} \hat{\mathbf{u}}\right)}{{ }^{n} \beta}\right|\left|1-\frac{{ }^{n} \beta}{{ }^{n} \gamma}\right| .
$$

As $\mathbf{u} \in \mathscr{H} \mathscr{R}_{\alpha}{ }^{d},\|\|^{n} \hat{\mathbf{u}} \|_{\infty}$ is in $\mathrm{O}(\alpha)$ and $\left\langle\left\|^{n} \mathrm{G}\right\|_{\infty}\right\rangle$ is in $\mathrm{O}\left(\beta^{2} \div \alpha\right)$, the ratio ${ }^{n} \mathrm{G}\left({ }^{n} \hat{\mathbf{u}}\right) /{ }^{n} \beta$ is in $\mathrm{O}(\beta)$. Moreover, by hypothesis, the ratio ${ }^{n} \beta /{ }^{n} \gamma$ tends toward 1 . Therefore, the difference between $\left({ }^{n} \mathrm{G}^{n} \hat{\mathbf{u}}\right) \div{ }^{n} \beta$ and ${ }^{n} g\left({ }^{n} \hat{\mathbf{u}}\right)$ is in $\mathrm{o}(\beta)$, that is, $\left\langle\left({ }^{n} \mathrm{G}^{n} \hat{\mathbf{u}}\right) \div{ }^{n} \beta\right\rangle={ }_{\beta}\left\langle{ }^{n} g\left({ }^{n} \hat{\mathbf{u}}\right)\right\rangle$.

Conversely, we assume a sequence of QLTs $\left\langle{ }^{n} g\right\rangle$ with scales ${ }^{n} \gamma$ such that $\left\langle{ }^{n} \gamma\right\rangle={ }_{\beta} \beta,\left\langle\left\|{ }^{n} \mathrm{G}\right\|_{\infty}\right\rangle \in \mathrm{O}\left(\beta^{2} \div \alpha\right)$ and, for any $\mathbf{u} \in \mathscr{H} \mathscr{R}_{\alpha}{ }^{d}$, there exists $\hat{\mathbf{u}}$ such that $\ell(\mathbf{u})=$ $\left[\left\langle{ }^{n} g\left({ }^{n} \hat{\mathbf{u}}\right)\right\rangle\right]$. Let ${ }^{n} \mathrm{G}$ be the matrix of the canonical representative of ${ }^{n} g$. Since, as shown in the first part of the proof, the sequences $\left\langle\left({ }^{n} \mathrm{G}^{n} \hat{\mathbf{u}}\right) \div{ }^{n} \beta\right\rangle$ and $\left\langle{ }^{n} g\left({ }^{n} \hat{\mathbf{u}}\right)\right\rangle$ are equivalent for the relation $=_{\beta}$, we derive that, for any $\mathbf{u}$ there exists $\hat{\mathbf{u}}$ such that

$$
\ell(\mathbf{u})=\left[\left\langle\left({ }^{n} \mathrm{G}^{n} \hat{\mathbf{u}}\right) \div{ }^{n} \beta\right\rangle\right] .
$$

Actually, since $\left\langle\left\|{ }^{n} \mathrm{G}\right\|_{\infty}\right\rangle \in \mathrm{O}\left(\beta^{2} \div \alpha\right)$, the latter equality is true for any representative of $\mathbf{u}$.

We are now able to prove the linearity of $\ell$.

$$
\begin{aligned}
\ell(\mathbf{u}+\mathbf{v}) & =\left[\left\langle{ }^{n} \mathrm{G}\left({ }^{n} \hat{\mathbf{u}}+{ }^{n} \hat{\mathbf{v}}\right) \div{ }^{n} \beta\right\rangle\right] \\
& =\left[\left\langle\left({ }^{n} \mathrm{G}\left({ }^{n} \hat{\mathbf{u}}\right)+{ }^{n} \mathrm{G}\left({ }^{n} \hat{\mathbf{v}}\right)\right) \div{ }^{n} \beta\right\rangle\right] \\
& =\left[\left\langle{ }^{n} \mathrm{G}\left({ }^{n} \hat{\mathbf{u}}\right) \div{ }^{n} \beta+{ }^{n} \mathrm{G}\left({ }^{n} \hat{\mathbf{u}}\right) \div{ }^{n} \beta+{ }^{n} R\right\rangle\right],
\end{aligned}
$$

where ${ }^{n} R \in\{0,1\}{ }^{d^{\prime}}$. Thus,

$$
\begin{aligned}
\ell(\mathbf{u}+\mathbf{v}) & =\left[\left\langle{ }^{n} \mathrm{G}\left({ }^{n} \hat{\mathbf{u}}\right) \div{ }^{n} \beta\right\rangle\right]+\left[\left\langle{ }^{n} \mathrm{G}\left({ }^{n} \hat{\mathbf{u}}\right) \div{ }^{n} \beta\right\rangle\right] \\
& =\ell(\mathbf{u})+\ell(\mathbf{v}) .
\end{aligned}
$$

Alike, we prove that $\ell\left(\lambda \times_{\alpha} \mathbf{u}\right)=\psi_{\beta} \circ \varphi_{\alpha}(\lambda) \times_{\beta} \ell(\mathbf{u})$ for any $\lambda \in \mathscr{H} \mathscr{R}_{\alpha}$. On the one hand, from the definition of the product $\times_{\alpha}$, we have $\lambda \times_{\alpha} \mathbf{u}=\left[\left\langle{ }^{n} \hat{\lambda}^{n} \hat{\mathbf{u}} \div{ }^{n} \alpha\right\rangle\right]$. Thereby,

$$
\ell\left(\lambda \times{ }_{\alpha} \mathbf{u}\right)=\left[\left\langle{ }^{n} \mathrm{G}\left({ }^{n} \hat{\lambda}^{n} \hat{\mathbf{u}} \div{ }^{n} \alpha\right) \div{ }^{n} \beta\right\rangle\right] .
$$

Then, picking a representative $a$ in $\ell\left(\lambda \times_{\alpha} \mathbf{u}\right)$,

$$
\begin{aligned}
a & ={ }_{\beta}\left\langle{ }^{n} \mathrm{G}\left({ }^{n} \hat{\lambda}^{n} \hat{\mathbf{u}} \div{ }^{n} \alpha\right) \div{ }^{n} \beta\right\rangle \\
& ={ }_{\beta}\left\langle\frac{{ }^{n} \hat{\lambda}}{{ }^{n} \alpha^{n} \beta}{ }^{n} \mathrm{G}\left({ }^{n} \hat{\mathbf{u}}\right)+O\left(\frac{\beta}{\alpha}\right)\right\rangle \\
& ={ }_{\beta}\left\langle\frac{{ }^{n} \hat{\lambda}}{{ }^{n} \alpha^{n} \beta}{ }^{n} \mathrm{G}\left({ }^{n} \hat{\mathbf{u}}\right)\right\rangle .
\end{aligned}
$$

On the other hand,

$$
\psi_{\beta} \circ \varphi_{\alpha}(\lambda) \times_{\beta} \ell(\mathbf{u})=\left[\left\lfloor\beta \frac{\hat{\lambda}}{\alpha}\right\rfloor\right] \times_{\beta}\left[\left\langle{ }^{n} \mathrm{G}\left({ }^{n} \hat{\mathbf{u}}\right) \div{ }^{n} \beta\right\rangle\right] .
$$

Picking a representative $b$ in $\psi_{\beta} \circ \varphi_{\alpha}(\lambda) \times_{\beta} \ell(\mathbf{u})$,

$$
\begin{aligned}
b & =\beta\left\langle\left(\left\lfloor\frac{{ }^{n} \beta^{n} \hat{\lambda}}{{ }^{n} \alpha}\right\rfloor\left({ }^{n} \mathrm{G}\left({ }^{n} \hat{\mathbf{u}}\right) \div{ }^{n} \beta\right)\right) \div{ }^{n} \beta\right\rangle \\
& ={ }_{\beta}\left\langle\frac{{ }^{n} \hat{\lambda}}{{ }^{n} \alpha^{n} \beta}{ }^{n} \mathrm{G}\left({ }^{n} \hat{\mathbf{u}}\right)+\mathrm{O}\left(\frac{{ }^{n} \lambda}{{ }^{n} \alpha}\right)+\mathrm{O}\left(\frac{{ }^{n} \mathrm{G}\left({ }^{n} \hat{\mathbf{u}}\right)}{\left({ }^{n} \boldsymbol{\beta}\right)^{2}}\right)+\mathrm{O}(1)\right\rangle
\end{aligned}
$$


From the hypotheses, we derive that $\left\langle{ }^{n} \lambda\right\rangle \in \mathrm{O}\left(\left\langle{ }^{n} \alpha\right\rangle\right)$ and $\left\langle{ }^{n} \mathrm{G}\left({ }^{n} \hat{\mathbf{u}}\right)\right\rangle \in \mathrm{O}\left(\left\langle{ }^{n} \beta^{2}\right\rangle\right)$. Thus,

$$
\begin{aligned}
b & ={ }_{\beta}\left\langle\frac{{ }^{n} \hat{\lambda}}{{ }^{n} \alpha^{n} \beta}{ }^{n} \mathrm{G}\left({ }^{n} \hat{\mathbf{u}}\right)+\mathrm{O}(1)\right\rangle \\
& ={ }_{\beta}\left\langle\frac{{ }^{n} \hat{\lambda}}{{ }^{n} \alpha^{n} \beta}{ }^{n} \mathrm{G}\left({ }^{n} \hat{\mathbf{u}}\right)\right\rangle .
\end{aligned}
$$

Therefore $a={ }_{\beta} b$, that is, $\ell\left(\lambda \times_{\alpha} \mathbf{u}\right)=\psi_{\beta} \circ \varphi_{\alpha}(\lambda) \times_{\beta}$ $\ell(\mathbf{u})$. Eventually, we conclude that $\ell$ is an $\Omega$-LT.

In Sec. 3.2, we have shown that sequences of QLTs arise from the $\Omega$-LTs between $\mathscr{H} \mathscr{R}_{\alpha}{ }^{d}$ and $\mathscr{H} \mathscr{R}_{\beta}{ }^{{ }^{\prime}}$ and in Sec. 3.1. it was established that those $\Omega$-LTs are in correspondance with the Euclidean linear maps. Thereby, we can achieve the $\Omega$-arithmetization scheme for the Euclidean linear maps. This is the purpose of the next section.

\subsection{Convergence}

Corollary 1 establishes a pointwise convergence property of some sequences of maps-involving $\Omega$-LTs - toward the Euclidean linear transformations. The main result of this section, Theorem 2, enforces the latter result by showing that the convergence is uniform on any compact subset of $\mathbb{R}^{n}$ and by describing the convergence speed. Furthermore, rather than appealing to $\Omega$-LT, the result is now expressed in terms of sequences of QLTs.

Before stating and proving our theorem, let us assess the relations between Euclidean linear maps, QLTs and $\Omega$-LT through a new commutative diagram (Fig 4). Besides, in the same way we defined in Sec. 3.1 the maps $\xi_{\gamma}^{\mathrm{HR}}: \mathbb{R}^{e} \rightarrow \mathscr{H} \mathscr{R}_{\gamma}{ }^{e}$ and $\chi_{\gamma}^{\mathrm{HR}}: \mathscr{H} \mathscr{R}_{\gamma}{ }^{e} \rightarrow \mathbb{R}^{e}(e \geq 1)$, we now define, for any infinitely large number $\omega$, the maps $\xi_{\omega}^{\mathbb{Z}}: \mathbb{R}^{e} \rightarrow\langle Z\rangle_{\omega}{ }^{e}$ and $\chi_{\omega}^{\mathbb{Z}}:\langle\mathbb{Z}\rangle_{\omega}{ }^{e} \rightarrow \mathbb{R}^{e}$ by $\xi_{\omega}^{\mathbb{Z}}(\mathbf{x})=\lfloor\omega \mathbf{x}\rfloor$ and $\chi_{\omega}^{\mathbb{Z}}\left(\left\langle{ }^{n} p\right\rangle\right)=\limsup \left\langle{ }^{n} p /{ }^{n} \omega\right\rangle$. These maps have the same properties as $\xi_{\gamma}^{\mathrm{HR}}$ and $\chi_{\gamma}^{\mathrm{HR}}: \xi_{\gamma}^{\mathbb{Z}}$ is an injective map and $\chi_{\gamma}^{\mathbb{Z}} \circ \xi_{\gamma}^{\mathbb{Z}}$ is the identity map of $\mathbb{R}^{e}$. Furthermore, the restriction of $\xi_{\gamma}^{\mathbb{Z}} \circ \chi_{\gamma}^{\mathbb{Z}}$ to $\xi_{\gamma}^{\mathbb{Z}}\left(\mathbb{R}^{e}\right)$ is the identity map of $\xi_{\gamma}^{\mathbb{Z}}\left(\mathbb{R}^{e}\right)$. Then, the linear subspace $\mathbf{y}=f(\mathbf{x})$ of $\mathbb{R}^{2} \times \mathbb{R}^{2}$, which perfectly defines the linear transformation $f$, is in one-to-one correspondence with the sequence of quasiplanes $\left\langle\mathbf{y}={ }^{n} \ell(\mathbf{x})\right\rangle$ of $\mathbb{Z}^{2} \times \mathbb{Z}^{2}$, providing a multiscale integer model of the plane $\mathbf{y}=f(\mathbf{x})$ for the sequence of scales $\left\langle\left({ }^{n} \alpha,{ }^{n} \beta\right)\right\rangle$.

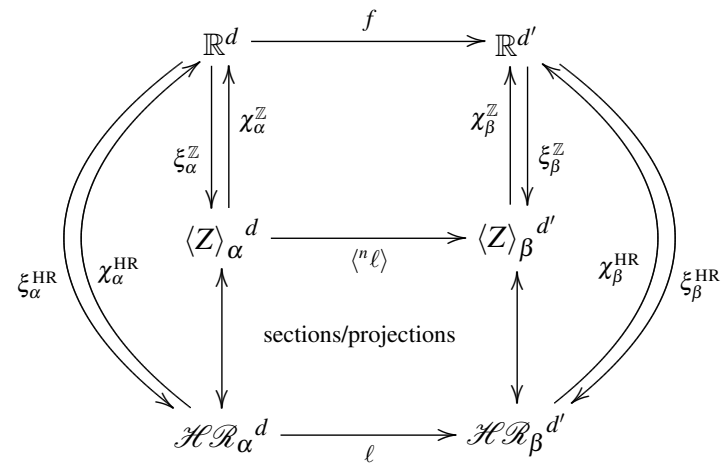

Figure 4: The skeleton of the $\Omega$-arithmetization scheme of a Euclidean linear map $f$. The $\Omega$-LT $\ell$ has for matrix the Harthong-Reeb version (over the ring $\mathscr{H} \mathscr{R}_{\omega}$ where $\omega=\beta^{2} \div \alpha$ ) of the matrix of $f$. The sections of the $\Omega$ LT for the involved equivalence relations give raise to sequences of QLTs $\left\langle{ }^{n} \ell\right\rangle$ which uniformly converge, up to a rescaling, toward the linear map $f$.

Theorem 2. Let $f: \mathbb{R}^{d} \rightarrow \mathbb{R}^{d^{\prime}}$ be a linear transformation, $F$ its matrix and $K \subset \mathbb{R}^{d}$ a compact set. Given $\alpha$, $\beta, \omega$ three infinitely large numbers such that $\beta^{2} \sim \alpha \omega$, we consider the sequence $\left\langle{ }^{n} g\right\rangle$ where ${ }^{n} g$ is the $Q L T$ whose canonical representation is $\left({ }^{n} \beta,\left\lfloor{ }^{n} \omega F\right\rfloor, \mathbf{0}\right)$. Then, for any $\mathbf{x} \in K$,

$$
\begin{aligned}
\| f(\mathbf{x}) & -\frac{{ }^{n} g\left(\left\lfloor{ }^{n} \alpha \mathbf{x}\right\rfloor\right)}{{ }^{n} \beta} \|_{\infty} \\
& \leq \frac{\|F\|_{\infty}}{{ }^{n} \alpha}+\frac{\left(\|F\|_{\infty}+1+d\right)\left(\|\mathbf{x}\|_{\infty}+1\right)}{{ }^{n} \boldsymbol{\omega}}+\frac{1}{{ }^{n} \beta} .
\end{aligned}
$$

Proof. We set $\mathbf{p}={ }^{n}\left(\xi_{\alpha}^{\mathbb{Z}}(\mathbf{x})\right)=\left\lfloor{ }^{n} \alpha \mathbf{x}\right\rfloor$ and $\mathrm{G}=$ ${ }^{n}\left(\xi_{\omega}^{\mathbb{Z}}(\mathrm{F})\right)=\left\lfloor{ }^{n} \omega \mathrm{F}\right\rfloor$. Moreover, we write $\alpha, \beta, \omega$ instead of ${ }^{n} \alpha,{ }^{n} \beta$ and ${ }^{n} \omega$. The column matrices of the vectors $\mathbf{p}$ and $\mathbf{x}$ are noted $\mathrm{P}$ and $\mathrm{X}$ respectively. Then, according to the definition of ${ }^{n} g$, the column matrix of the vector $f(\mathbf{x})-{ }^{n} g(\mathbf{p}) / \beta$ is

$$
\mathrm{FX}-\frac{1}{\beta}\left\lfloor\frac{1}{\beta} \mathrm{GP}\right\rfloor .
$$

We split this vector in four parts whose norms will then 
be bounded from above.

$$
\begin{aligned}
& \mathrm{FX}-\frac{1}{\beta}\left\lfloor\frac{1}{\beta} \mathrm{GP}\right\rfloor \\
& =\left(\mathrm{FX}-\frac{1}{\alpha} \mathrm{FP}\right)+\left(\frac{1}{\alpha} \mathrm{FP}-\frac{1}{\beta}\left\lfloor\frac{1}{\beta} \mathrm{GP}\right\rfloor\right) \\
& =\frac{1}{\alpha} \mathrm{F}(\alpha \mathrm{X}-\mathrm{P})+\frac{1}{\alpha \omega}(\omega \mathrm{F}-\mathrm{G}) \mathrm{P}+ \\
& \quad\left(\frac{1}{\alpha \omega} \mathrm{GP}-\frac{1}{\beta}\left\lfloor\frac{1}{\beta} \mathrm{GP}\right\rfloor\right) \\
& =\frac{1}{\alpha} \mathrm{F}(\alpha \mathrm{X}-\mathrm{P})+\frac{1}{\alpha \omega}(\omega \mathrm{F}-\mathrm{G}) \mathrm{P}+ \\
& \quad\left(\frac{\beta^{2}}{\alpha \omega}-1\right)\left(\frac{1}{\beta^{2}} \mathrm{GP}\right)+\frac{1}{\beta}\left(\frac{1}{\beta} \mathrm{GP}-\left\lfloor\frac{1}{\beta} \mathrm{GP}\right\rfloor\right)
\end{aligned}
$$

The four terms of the latter sum are now bounded from above in infinite norm.

- Because $\mathrm{P}=\lfloor\alpha \mathrm{X}\rfloor$, one has

$$
\begin{aligned}
& \|(1 / \alpha) \mathrm{F}(\alpha \mathrm{X}-\mathrm{P})\|_{\infty} \\
& \quad \leq(1 / \alpha)\|\mathrm{F}\|_{\infty} \|\left(\alpha \mathrm{X}-\mathrm{P}\left\|_{\infty} \leq(1 / \alpha)\right\| \mathrm{F} \|_{\infty}\right.
\end{aligned}
$$

- the coefficients of the matrix $\omega \mathrm{F}-\mathrm{G}$ are fractional parts: $(\omega \mathrm{F}-\mathrm{G})_{i, j}=\omega \mathrm{F}_{i, j}-\left\lfloor\omega \mathrm{F}_{i, j}\right\rfloor$. Then,

$$
\begin{aligned}
& \|(1 / \alpha \omega)(\omega \mathrm{F}-\mathrm{G}) \mathrm{P}\|_{\infty} \\
& \leq(1 / \alpha \omega)\|\omega \mathrm{F}-\mathrm{G}\|_{\infty}\|\mathrm{P}\|_{\infty}<(d / \alpha \omega)\|\mathrm{P}\|_{\infty} .
\end{aligned}
$$

Since $\|\mathbf{p}\|_{\infty}<\alpha\|\mathbf{x}\|_{\infty}+1 \leq \alpha\left(\|\mathbf{x}\|_{\infty}+1\right)$, it follows that $\|(1 / \alpha \omega)(\omega \mathrm{F}-\mathrm{G}) \mathrm{P}\|_{\infty}$ is less than $(d / \omega)\left(\|\mathbf{x}\|_{\infty}+1\right)$

- By definition of $\omega, \beta^{2}-\alpha \omega<\alpha$. Furthermore, $\|\mathrm{G}\|_{\infty}<\omega\left(\|\mathrm{F}\|_{\infty}+1\right)$ and $\|\mathbf{p}\|_{\infty}<\alpha\left(\|\mathbf{x}\|_{\infty}+1\right)$. Then, the term $\left\|\left(\left(\beta^{2} / \alpha \omega\right)-1\right)\left(\left(1 / \beta^{2}\right) \mathrm{GP}\right)\right\|_{\infty}$ is bounded from above by $(1 / \omega)\left(\|\mathrm{F}\|_{\infty}+1\right)\left(\|\mathbf{x}\|_{\infty}+\right.$ $1)$.

- $(1 / \beta)\|(1 / \beta) \mathrm{GP}-\lfloor(1 / \beta) \mathrm{GP}\rfloor\|_{\infty} \quad$ is clearly bounded from above by $1 / \beta$.

Putting together all these upper bounds, we obtain the desired inequality.

\section{Conclusion}

Via a constructive version of the real numbers, the Harthong-Reeb line, we have obtained a multi-scale arithmetization scheme of the Euclidean affine transformations. In this framework, an infinite sequence of quasilinear transformations is the translation of a single Euclidean affine map. Depending, on the choices of the infinitely large numbers $\alpha, \beta, \omega$, the obtained sequence can be more or less obvious. Nevertheless, the merit of this approach is to bring an insight into the properties of discrete computations of affine maps and to open a new field of study. Indeed, it is well known that computer affine transformation implementations are not one-to-one, do not preserve fixed points, topology and so on. But the Harthong-Reeb model show that such implementations operate on sections of equivalence classes instead of equivalence classes. Then, for instance, a Euclidean vector may have a singular preimage while its section on a computer have a non-singular preimage which contains the points of the equivalence class that are exposed by the section (preimage structures are studied in the papers [13 15] among others). Then, the next task in the proposed framework is to revisit the properties of the quasilinear transformations under this multi-scale interpretation derived from the Harthong-Reeb line.

\section{References}

[1] Blot, V., Cœurjolly, D.: Quasi-affine transformation in higher dimension. In: DGCI, LNCS, vol. 5810, pp. 493-504 (2009). URL http://dx.doi.org/ 10.1007/978-3-642-04397-0_42

[2] Chollet, A.: Non classical formalisms for the computing treatment of the topology and the discrete geometry. Ph.D. thesis, Université de La Rochelle (2010). URL https://tel. archives-ouvertes.fr/tel-00579781

[3] Chollet, A., Wallet, G., Andres, E., Fuchs, L., Largeteau-Skapin, G., Richard, A.: Omegaarithmetization of ellipses. In: CompIMAGE, pp. 24-35 (2010). URL http://dx.doi.org/10. 1007/978-3-642-12712-0_3 
[4] Chollet, A., Wallet, G., Fuchs, L., Andres, A., Largeteau-Skapin, G.: Omega-arithmetization: A discrete multi-resolution representation of real functions. In: IWCIA, pp. 316-329 (2009). URL http://dx.doi.org/10.1007/ 978-3-642-10210-3_25

[5] Chollet, A., Wallet, G., Fuchs, L., Andres, E., Largeteau-Skapin, G.: Foundational aspects of multiscale digitization. Theor. Comput. Sci. 466, 2-19 (2012). DOI 10.1016/j.tcs.2012.07. 026. URL http://dx.doi.org/10.1016/j. tcs.2012.07.026

[6] Chollet, A., Wallet, G., Fuchs, L., Largeteau-Skapin, G., Andres, E.: Insight in discrete geometry and computational content of a discrete model of the continuum. Pattern Recognition 42(10), 2220-2228 (2009). URL http://dx.doi.org/10.1016/j . patcog.2008.12.005

[7] Coeurjolly, D., Blot, V., Jacob-Da Col, M.A.: Quasiaffine transformation in 3-d: Theory and algorithms. In: IWCIA, LNCS, vol. 5852, pp. 6881 (2009). URL http://dx.doi.org/10.1007/ 978-3-642-10210-3_6

[8] Diener, F., Reeb, G.: Analyse non standard. Hermann (1989)

[9] Fuchs, L., Largeteau-Skapin, G., Wallet, G., Andres, E., Chollet, A.: A first look into a formal and constructive approach for discrete geometry using nonstandard analysis. In: DGCI, LNCS, pp. 2132 (2008). URL http://dx.doi.org/10.1007/ 978-3-540-79126-3_4

[10] Harthong, J.: Une théorie du continu. In: Les mathématiques non standard, pp. 307-329 (1989)

[11] Jacob, M.A.: Applications quasi-affines. Ph.D. thesis, Université Louis Pasteur, Strasbourg (1993)

[12] Jacob-Da Col, M.A.: Applications quasi-affines et pavages du plan discret. Theoretical Computer Science 259(1-2), 245-269 (2001). URL https:// dpt-info.u-strasbg.fr/ jacob
[13] Jacob-Da Col, M.A., Mazo, L.: nD quasi-affine transformations. In: DGCI, LNCS, pp. 337348 (2016). URL http: //dx . doi .org/10 . 1007/ 978-3-319-32360-2_26

[14] Jacob-Da Col, M.A., Reveillès, J.P.: Gaussian numeration systems. In: DGCI, LNCS, pp. 37-47 (1995). URL https://dpt-info.u-strasbg. $\mathrm{fr} /{ }^{\sim}$ jacob

[15] Jacob-Da Col, M.A., Tellier, P.: Quasi-linear transformations and discrete tilings. Theoretical Computer Science 410(21), 2126-2134 (2009). URL http://dx.doi.org/10.1016/j . tcs.2009.01.032

[16] Laugwitz, D.: $\Omega$-calculus as a generalization of field extension: An alternative approach to nonstandard analysis. In: Nonstandard Analysis - Recent developments, LNM, pp. 144-155 (1983)

[17] Magaud, N., Chollet, A., Fuchs, L.: Formalizing a discrete model of the continuum in coq from a discrete geometry perspective. Annals of Mathematics and Artificial Intelligence 74(3-4), 309332 (2015). URL http://dx.doi.org/10.1007/ s10472-014-9434-6

[18] Nehlig, P.: Applications quasi-affines : pavages par images réciproques. Theoretical Computer Science 156(1\&2), 1-38 (1996). URL http://dx.doi. org/10.1016/0304-3975(95) 00040-2

[19] Reveillès, J.P.: Géométrie discrète, calcul en nombres entiers et algorithmique. Ph.D. thesis, Université Louis Pasteur, Strasbourg (1991)

[20] Reveillès, J.P., Richard, D.: Back and forth between continuous and discrete for the working computer scientist. Annals of Mathematics and Artificial Intelligence 16(1), 89-152 (1996). URL https: //doi.org/10.1007/BF02127796

[21] Schmieden, C., Laugwitz, D.: Eine erweiterung der infinitesimalrechnung. Mathematische Zeitschrift 69, 1-39 (1958) 